\title{
Auteurs
}

\section{Nordine KERKAR}

Actuellement responsable du groupe Neutronique et Physique des cœurs du Service d'études du parc thermique et nucléaire à Électricité De France. Après des études doctorales consacrées à l'industrialisation d'une nouvelle méthode 3D temps réel de pilotage des réacteurs nucléaires, menées à AREVA-NP en collaboration avec le Commissariat à I'énergie atomique, il rejoint la Direction recherche et développement du Groupe EDF. II y conduit des travaux portant sur la modélisation des réflecteurs des cœurs REP et contribue à la mise au point des outils de calculs 3D couplés neutronique-thermohydraulique et aux méthodes de pénalisation associées adaptées aux études d'accidents. II est l'auteur d'une méthode originale d'équivalence multigroupe-multisolveur adaptée aux techniques modernes de calcul des réacteurs nucléaires et des réacteurs expérimentaux. Il rejoint ensuite I'Unité nationale d'ingénierie du parc en exploitation, où il prend la responsabilité des applications industrielles utilisées pour le suivi, les essais et la protection des cœurs en exploitation. En parallèle, il a donné des cours sur les méthodes d'éléments finis à I'École centrale de Paris et a assuré pendant quatre ans l'enseignement du module cœurcombustible du Génie Atomique à I'Institut national des sciences et techniques nucléaires de Saclay.

\section{Philippe PAULIN}

Ingénieur à Électricité De France, ancien élève de SUPELEC, il travaille depuis 1981 dans le domaine de l'exploitation des cœurs. Son expérience s'étend aux deux filières RNR en tant qu'ingénieur au Service physique du cœur du CNPE de Creys-Malville et REP comme responsable du service Méthodes de la Branche Combustible de l'Unité nationale d'ingénierie du parc en exploitation. Il est actuellement attaché technique au Groupe exploitation cœur combustible de I'Unité d'ingénierie d'exploitation (UNIE) de la Division production nucléaire (DPN). En parallèle, il a assuré l'enseignement du module cœurcombustible du cours de Génie Atomique à l'Institut national des sciences et techniques nucléaires. 
This page intentionally left blank 